\title{
Numerical Analysis of Melting/Solidification Phenomena Using a Moving Boundary Analysis Method X-FEM
}

\author{
Akihiro Uchibori and Hiroyuki Ohshima \\ Japan Atomic Energy Agency \\ Japan
}

\section{Introduction}

Melting/solidification or dissolution/precipitation phenomena appear in several candidate technologies for fast reactor fuel cycle. The fuel cycle consists of reprocessing of spent fuels and fuel fabrication. Combination of the advanced aqueous reprocessing system and the simplified pelletizing fuel fabrication system is a most promising concept for recycling mixed oxide fuels (Sagayama, 2007; Funasaka \& Itoh, 2007). The advanced aqueous reprocessing includes the process of dissolution of sheared spent fuels in a nitric acid. The most part of uranium is recovered from the dissolved solution by crystallization. There are dissolution/precipitation phenomena also in the metal electro-refining pyrochemical reprocessing, which is a candidate technology for reprocessing of metal fuels (Kofuji, 2010). In this technology, uranium and plutonium are recovered from spent metal fuels by electrolysis. A metal fuel slug is formed by the injection casting method. In this method, an injected molten fuel alloy solidifies in the mold. Melting/solidification phenomena appear also in the process of high-level radioactive wastes disposal (Kozaka \& Tominaga, 2005). High-level radioactive wastes are fed into a melter and mixed with a glass melt. The molten mixture is subsequently cooled in a canister. Melting/solidification or dissolution/precipitation phenomena have been applied not only in the above-mentioned technologies but also in many engineering fields.

Numerical analysis considering melting/solidification or dissolution/precipitation is very useful to evaluate operating conditions and designs of equipment configuration in these technologies. However, there is a difficulty that a moving solid-liquid interface exists in such phase change problems. Reproducing move of the solid-liquid interface in numerical analysis is a challenging problem. The existing numerical analysis methods for melting/solidification problems can be classified into two groups: a moving mesh method and an enthalpy method. In the former group, an analysis mesh is reconstructed during calculation so that nodes overlap with the solid-liquid interface. Independent energy equations of the each phase are coupled by an appropriate boundary condition at the solidliquid interface. Lynch \& O'Neill (1981) developed a moving mesh finite element method, which is a typical method of the former group. Sampath \& Zabaras (1999) applied the moving mesh finite element method to a three-dimensional directional solidification 
problem. A variable space network method developed by Murray \& Landis (1959) and a front tracking ALE (Arbitrary Lagrangian Eulerian) method developed by Jaeger \& Carin (1994) are also classified as the former group. While the moving mesh methods give high accuracy in predicting an interface position, there is the drawback that the algorithm of remeshing is complicated especially in the case of multi-dimensional problems. When a shape of the solid-liquid interface becomes complex, we have to pay attention to deformation of elements. Therefore, the moving mesh methods are frequently used for directional melting/solidification problems. The latter group (i.e. an enthalpy method) introduces an artificial heat capacity containing latent heat for phase change. This enables us to eliminate a boundary condition at the solid-liquid interface. The enthalpy method requires only a single energy equation. Numerical analysis using the enthalpy method may be found in the paper by Comini et al. (1974) or by Rolph \& Bathe (1982). The enthalpy method is quite popular for multi-dimensional problems because re-meshing is not required. However, there is also the drawback that isothermal phase change phenomena cannot be modeled consistently. This is due to the inevitable assumption that phase change occurs within a certain range of temperature. Application of the above-mentioned two types of the methods is limited by their inherent drawbacks.

In application to the actual problems of the nuclear fuel cycle, a numerical analysis method needs to be applicable to multi-dimensional problems which involve complex move of the solid-liquid interface. A numerical analysis method using a fixed mesh can be simply applied to multi-dimensional problems even if they involves complex move of the interface. However, existence of a discontinuous temperature gradient at the solid-liquid interface complicates calculation of heat conduction and interface tracking in a fixed mesh. The eXtended Finite Element Method (X-FEM), which is a fixed-mesh-based-method, can overcome this difficulty. This method introduces an enriched finite element interpolation to represent the discontinuous temperature gradient in the element. The enriched finite element interpolation consists of a standard shape function and a signed distance function. This makes it possible to track the moving solid-liquid interface accurately without remeshing. The X-FEM has the advantages of both the moving mesh method and the enthalpy method. Moës et al. (1999) developed the X-FEM for arbitrary crack growth problems. Merle \& Dolbow (2002) and Chessa et al. (2002) applied the X-FEM to melting/solidification problems. Further, Chessa \& Belytschko (2003) simulated a two-phase flow problem involving bubble deformation successfully by using the X-FEM. These researches indicate that the X-FEM is widely applicable to moving interface problems.

The X-FEM is most effective as a tool to evaluate the melting/solidification or the dissolution/precipitation processes appearing in the nuclear fuel cycle. But the way to apply the X-FEM to phase change problems has not been fully elucidated. In this study, application of the X-FEM to melting/solidification problems was discussed. Formulation of the enriched finite element interpolation and construction of the finite element equation using it are reported below. The technique of quadrature and the method to solve the problems involving liquid flows were constructed in the present work. The numerical solutions of the example problems, i.e. a one-dimensional Stefan problem and solidification in a two-dimensional square corner, were compared to the existing solutions to verify validity of the proposed numerical method. Verification to the melting/solidification problem involving natural convection was also conducted. 


\section{Numerical methods}

\subsection{Governing equations}

We consider a melting/solidification problem shown in Fig. 1 . The domain $\Omega$ is divided into the solid region $\Omega_{s}$ and the liquid region $\Omega_{l}$. The boundary of the domain $\Omega$ and the interface between the solid region and the liquid region are denoted by $\Gamma$ and $\Gamma_{I}$, respectively. The governing equations for this problem are as follows:

$$
\begin{gathered}
\nabla \cdot \mathbf{u}=0 \\
\rho\left(\frac{\partial \mathbf{u}}{\partial t}+\mathbf{u} \cdot \nabla \mathbf{u}\right)=-\nabla p+\mu \nabla^{2} \mathbf{u}+\rho \mathbf{f} \\
\rho c_{p}\left(\frac{\partial T}{\partial t}+\mathbf{u} \cdot \nabla T\right)=\lambda \nabla^{2} T
\end{gathered}
$$

where $\mathbf{u}$ is the velocity vector, $\rho$ is the density, $t$ is the time, $p$ is the pressure, $\mu$ is the viscosity, $\mathbf{f}$ is the external force, $c_{p}$ is the specific heat, $T$ is the temperature and $\lambda$ is the thermal conductivity. Equations (1) and (2) are applied only to the liquid phase.

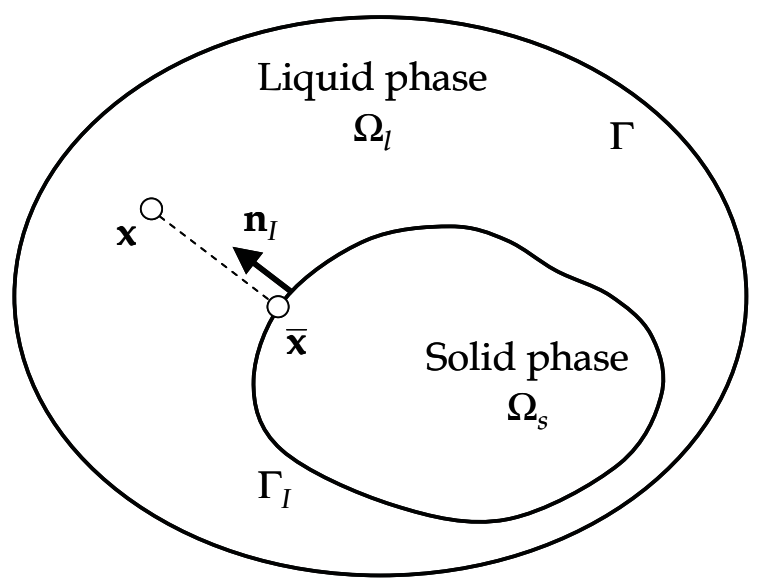

Fig. 1. Melting/solidification problem

A heat balance condition at the solid-liquid interface is written as

$$
\rho_{s} L V_{I}=\lambda_{s}\left[\frac{\partial T}{\partial n}\right]_{I s}-\lambda_{l}\left[\frac{\partial T}{\partial n}\right]_{I l}
$$

where $L$ is the latent heat for phase change, $V_{I}$ is the interface velocity (normal direction to the interface) and $n$ is the normal direction to the solid-liquid interface. Subscript $s$ stands for the solid, $l$ the liquid and $I$ the solid-liquid interface. Equation (4) means that the net heat flux at the solid-liquid interface is translated into the latent heat for phase change per unit 
time (see Fig. 2). The interface velocity can be obtained from Eq. (4). A temperature condition at the solid-liquid interface is given by

$$
T=T_{m}
$$

where $T_{m}$ is the melting temperature. Equation (5) is used as a constraint condition to Eq. (3), as described later. Melting/solidification problems appearing in the nuclear fuel area are basically described by Eq. (1) to (5).

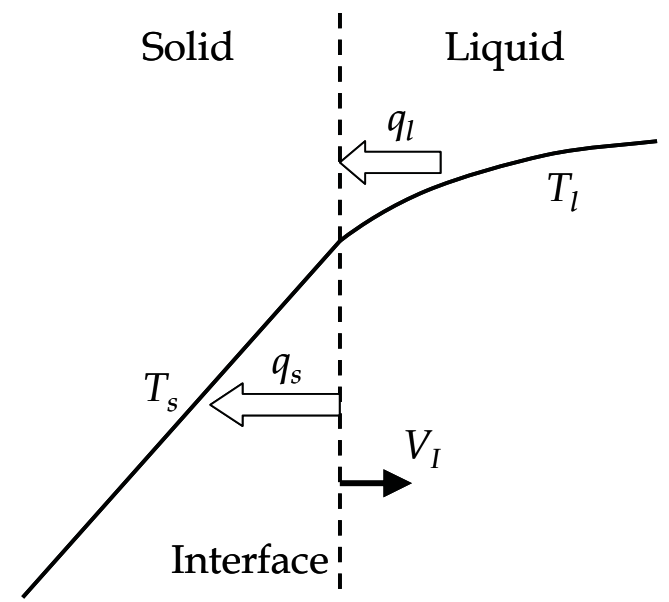

Fig. 2. Temperature profile and heat flux near solid-liquid interface

\subsection{Enriched finite element interpolation}

In melting/solidification problems, the temperature gradient is discontinuous at the solidliquid interface (see Fig. 2). A basic idea of the X-FEM is to introduce an enriched finite element interpolation to represent the discontinuous gradient of the temperature. In this section, we report the formulation described in the paper by Chessa et al. (2002) and present the temperature profile represented by the enriched finite element interpolation in the case of a two-node line element.

We define the element crossed by the solid-liquid interface as a fully enriched element. The node of the fully enriched element is defined as an enriched node. Figure 3 shows definition of the enriched element and the enriched node in the case of a one- or two-dimensional mesh. The enriched finite element interpolation is based on a standard interpolation:

$$
T(\mathbf{x}, t)=\sum_{i=1}^{n} N_{i}(\mathbf{x}) T_{i}(t)
$$

where $i$ is the node number, $n$ is the number of nodes, $N_{i}(\mathbf{x})$ is the shape function and $T_{i}(t)$ is the nodal value of the temperature:

$$
T_{i}(t)=T\left(\mathbf{x}_{i}, t\right)
$$


Enriched node

$\bigcirc$ Regular node

Interface

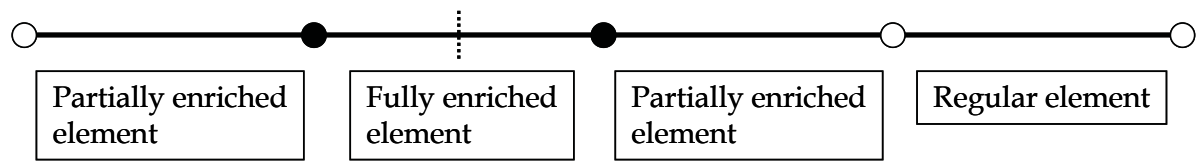

$1 \mathrm{D}$

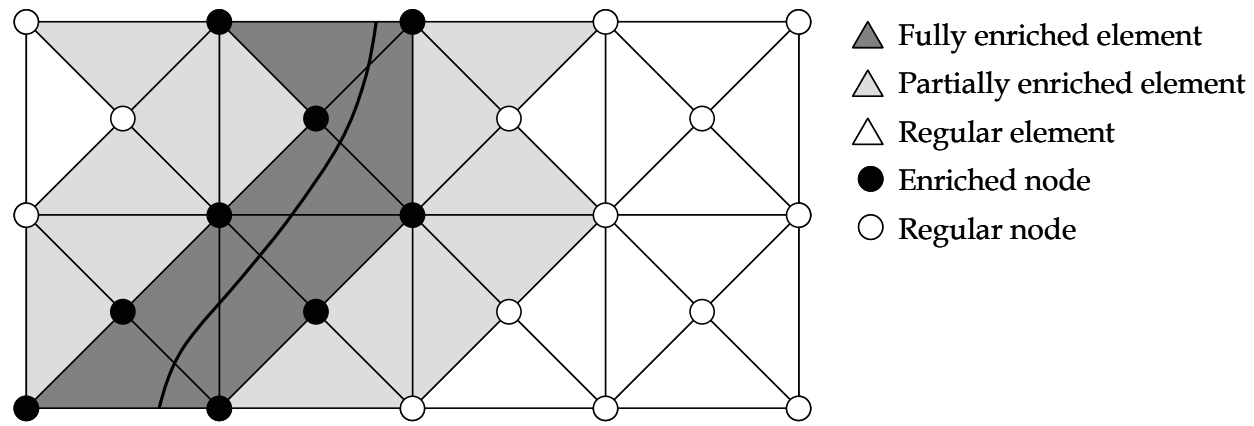

$2 \mathrm{D}$

Fig. 3. Definition of enriched elements and nodes

Figure 4(a) shows the temperature profile interpolated by Eq. (6) in the case of a two-node line element. To construct a discontinuous interpolation, we introduce a signed distance function $\phi(\mathbf{x}, t)$ :

$$
\phi(\mathbf{x}, t)=\min _{\overline{\mathbf{x}} \in \Gamma_{I}}\|\mathbf{x}-\overline{\mathbf{x}}\| \operatorname{sign}\left[\mathbf{n}_{I} \cdot(\mathbf{x}-\overline{\mathbf{x}})\right]
$$

where \|\| is the Euclidian norm, $\overline{\mathbf{x}}$ is the coordinate on the solid-liquid interface and $\mathbf{n}_{I}$ is the outward normal unit vector from the solid region (see Fig. 1). This definition means the shortest distance from $\mathbf{x}$ to the solid-liquid interface. The signed distance function varies as follows:

$$
\phi(\mathbf{x}, t) \begin{cases}<0 & \mathbf{x} \in \Omega_{s} \\ =0 & \mathbf{x} \in \Gamma_{I} \\ >0 & \mathbf{x} \in \Omega_{l}\end{cases}
$$

Here, we define an enrichment function $\psi_{i}(\mathbf{x}, t)$ :

$$
\psi_{i}(\mathbf{x}, t)=N_{i}(\mathbf{x})\left(|\phi(\mathbf{x}, t)|-\left|\phi\left(\mathbf{x}_{i}, t\right)\right|\right)
$$


Figure $4(\mathrm{~b})$ shows the profile of the enrichment function $\psi_{1}$ and $\psi_{2}$. As can be seen, the enrichment function is discontinuous at the solid-liquid interface. Figure 4(c) shows the profile of $\psi_{1}+\psi_{2}$. This summation becomes linear between the interface and the node. Adding the enrichment function to the standard interpolation function, we obtain the enriched finite element interpolation:

$$
T(\mathbf{x}, t)=\sum_{i=1}^{n} N_{i}(\mathbf{x}) T\left(\mathbf{x}_{i}, t\right)+\sum_{j=1}^{n_{e}} \psi_{j}(\mathbf{x}, t) a_{j}(t)
$$

where $n_{e}$ is the number of enriched nodes and $a_{j}(t)$ is the additional degrees of freedom. The temperature profile interpolated by Eq. (11) is shown in Fig. 4(d). While the two-node regular element has two degrees of freedom (i.e. the unknowns, $T_{1}$ and $T_{2}$ ), the fully enriched element has four degrees of freedom (i.e. the unknowns, $T_{1}, T_{2}, a_{1}$ and $a_{2}$ ). The additional degrees of freedom, $a_{1}$ and $a_{2}$, are decided so that the temperature at the solidliquid interface becomes the melting temperature. The way to constrain the condition of the temperature at the solid-liquid interface to the finite element equation is described in section 2.3.2. The gradient of the temperature is written as

$$
\begin{aligned}
\nabla T(\mathbf{x}, t) & =\sum_{i=1}^{n} \nabla N_{i}(\mathbf{x}) T\left(\mathbf{x}_{i}, t\right) \\
& +\sum_{j=1}^{n_{e}} a_{j}(t)\left[\nabla N_{j}(\mathbf{x})\left(|\phi(\mathbf{x}, t)|-\left|\phi\left(\mathbf{x}_{j}, t\right)\right|\right)+N_{j}(\mathbf{x}) \nabla\left(|\phi(\mathbf{x}, t)|-\left|\phi\left(\mathbf{x}_{j}, t\right)\right|\right)\right.
\end{aligned}
$$

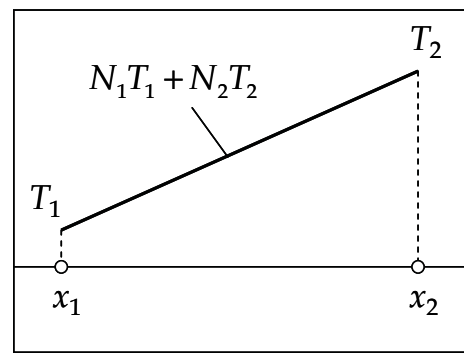

(a)

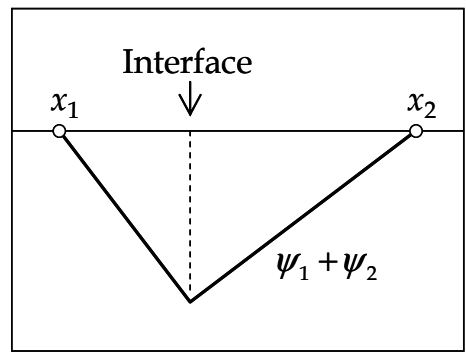

(c)

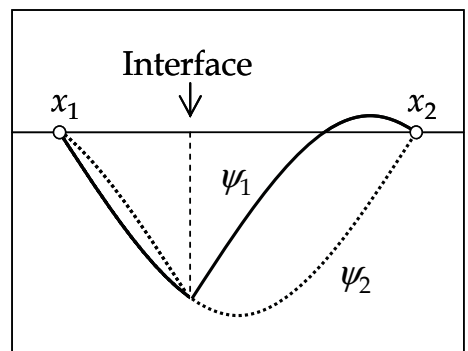

(b)

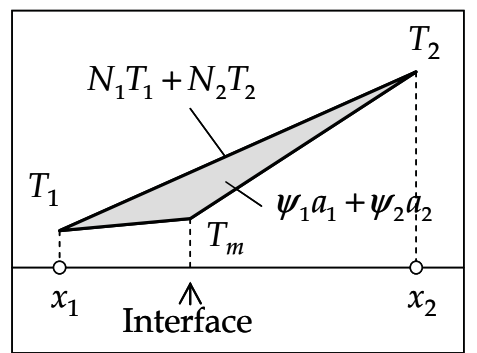

(d)

Fig. 4. Enriched finite element interpolation in two-node line element 
Not all of the nodes are enriched in the element adjacent to the fully enriched element. This partially enriched element is also shown in Fig. 3. If we apply the enriched interpolation defined as Eq. (11) to the partially enriched element, the unnecessary numerical value is added to the desired profile. This problem has been discussed in the paper by Chessa et al. (2003). We have used their method to avoid the incorrect interpolation.

\subsection{Finite element equation}

\subsubsection{Matrix form}

Based on the enriched finite element interpolation, i.e. Eq. (11), a finite element equation of the energy equation is derived here. For describing the finite element equation in matrix form, it is convenient to rewrite Eq. (11) as

$$
T(\mathbf{x}, t)=\overline{\mathbf{N}}(\mathbf{x}, t) \overline{\mathbf{T}}(t)
$$

where

$$
\begin{aligned}
\overline{\mathbf{N}}(\mathbf{x}, t) & =\left[\begin{array}{llllllll}
N_{1} & N_{2} & \cdots & N_{n} & \psi_{1} & \psi_{2} & \cdots & \psi_{n_{e}}
\end{array}\right] \\
\overline{\mathbf{T}}(t) & =\left[\begin{array}{llllllll}
T_{1} & T_{2} & \cdots & T_{n} & a_{1} & a_{2} & \cdots & a_{n_{e}}
\end{array}\right]^{T}
\end{aligned}
$$

The matrix form of Eq. (3) is

$$
\left(\frac{1}{\Delta t} \mathbf{M}^{n+1}+\mathbf{C}^{n+1}+\mathbf{K}^{n+1}\right) \overline{\mathbf{T}}^{n+1}=\frac{1}{\Delta t} \mathbf{M}^{n} \overline{\mathbf{T}}^{n}
$$

where $\mathbf{M}$ is the mass matrix, $\mathbf{C}$ is the coefficient matrix of the convection term and $\mathbf{K}$ is the coefficient matrix of the heat conduction term. These coefficient matrices consist of the shape function and the enrichment function. The mass matrix in the left hand side is written as

$$
\mathbf{M}^{n+1}=\left[\begin{array}{ll}
\mathbf{M}_{1}^{n+1} & \mathbf{M}_{2}^{n+1} \\
\mathbf{M}_{3}^{n+1} & \mathbf{M}_{4}^{n+1}
\end{array}\right]
$$

where

$$
\begin{aligned}
& {\left[\mathbf{M}_{1}^{n+1}\right]_{i j}=\int_{\Omega} \rho c_{p} N_{i} N_{j} d \Omega} \\
& {\left[\mathbf{M}_{2}^{n+1}\right]_{i j^{\prime}}=\int_{\Omega} \rho c_{p} N_{i} \psi_{j^{\prime}}^{n+1} d \Omega} \\
& {\left[\mathbf{M}_{3}^{n+1}\right]_{i^{\prime} j}=\int_{\Omega} \rho c_{p} \psi_{i^{\prime}}^{n+1} N_{j} d \Omega} \\
& {\left[\mathbf{M}_{4}^{n+1}\right]_{i^{\prime} j^{\prime}}=\int_{\Omega} \rho c_{p} \psi_{i^{\prime}}^{n+1} \psi_{j^{\prime}}^{n+1} d \Omega} \\
& 1 \leq i \leq n, 1 \leq j \leq n, 1 \leq i^{\prime} \leq n_{e}, 1 \leq j^{\prime} \leq n_{e}
\end{aligned}
$$

The other coefficient matrices are given similarly. 


\subsubsection{Enforcement of interface temperature condition}

The interface temperature condition, i.e. Eq. (5), acts as a constraint condition to the finite element equation, i.e. Eq. (16). This constraint condition was enforced by the penalty method (Chessa et al., 2002). In the penalty method, a constrained problem is simply converted to an unconstrained one. By using Eqs. (10) and (11), Eq. (5) is rewritten as

$$
\sum_{i=1}^{n} N_{i} T_{i}-\sum_{j=1}^{n_{e}} N_{j}\left|\phi_{j}\right| a_{j}-T_{m}=0
$$

The penalty forcing term is

$$
\mathbf{F}_{p}=\beta \mathbf{G}^{T} \mathbf{G}^{n+1}-\beta \mathbf{G}^{T} \mathbf{c}
$$

where $\beta$ is the penalty number,

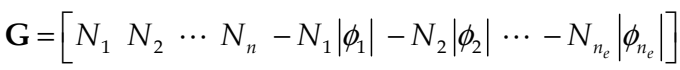

$$
\begin{aligned}
& \mathbf{c}=\left[T_{m}\right]
\end{aligned}
$$

and superscript $T$ stands for transpose of the matrix. Adding the global form of the penalty forcing term to the finite element equation, we obtain the final form:

$$
\left[\frac{1}{\Delta t} \mathbf{M}^{n+1}+\mathbf{C}^{n+1}+\mathbf{K}^{n+1}+\beta\left(\mathbf{G}^{n+1}\right)^{T} \mathbf{G}^{n+1}\right] \overline{\mathbf{T}}^{n+1}=\frac{1}{\Delta t} \mathbf{M}^{n} \overline{\mathbf{T}}^{n}+\beta\left(\mathbf{G}^{n+1}\right)^{T} \mathbf{c}
$$

In each of the iterative calculation step to solve Eq. (23), the penalty number is updated to be a value larger than that in the previous step. When the penalty number becomes a large value, $\mathbf{G} \overline{\mathbf{T}}^{n+1}-\mathbf{c}$ is enforced to be small.

It should be noted that the size of the global coefficient matrix changes with move of the solid-liquid interface in the case of multi-dimensional problem and the global coefficient matrix in Eq. (23) is non-symmetric. The Incomplete LU decomposition preconditioned BiConjugate Gradient (ILU-BCG) method was employed to solve Eq. (23).

\subsection{Gaussian quadrature for enriched elements}

The coefficient matrices in the finite element equation, i.e. Eq. (23), are calculated by using the Gaussian quadrature. Since the enrichment function is discontinuous (see Fig. 4), the standard quadrature method will lead to incorrect integration. In order to achieve correct integration in the fully enriched element, the element is divided into two sub-elements as shown in Fig. 5 (Chessa et al., 2002). In the present work, the method to implement the Gaussian quadrature to the sub-elements was formulated by introducing two reference coordinates. Since most problems in nuclear fuel area are multi-dimensional, a numerical technique needs to be applicable to multi-dimensional elements. Although formulation in the case of a two-node line element is described in this section, the method mentioned here can be simply applied to two- or three-dimensional analyses. 
$\times$ Quadrature point

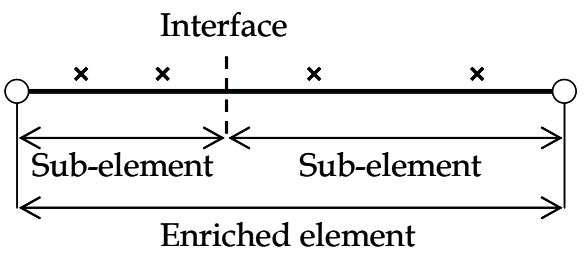

$1 \mathrm{D}$

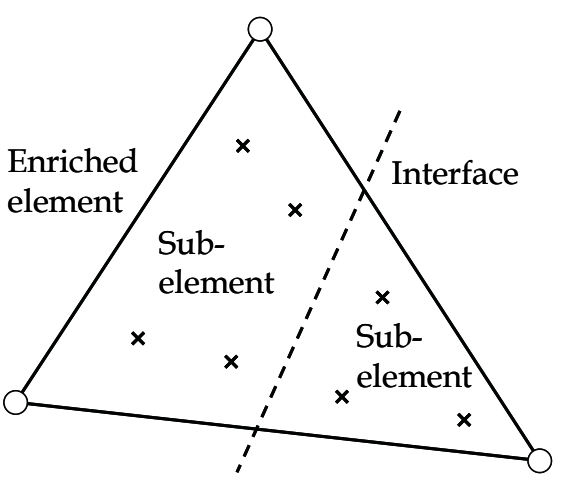

$2 \mathrm{D}$

Fig. 5. Sub-elements and quadrature points

The component of the mass matrix $\mathbf{M}$, i.e. $\int \rho c_{p} N_{i}(x) \psi_{j}(x) d x$, is divided into two integrations:

$$
\int_{\Omega_{e}} \rho c_{p} N_{i}(x) \psi_{j}^{n+1}(x) d x=\int_{\Omega_{e s}} \rho_{s} c_{p s} N_{i}(x) \psi_{j}^{n+1}(x) d x+\int_{\Omega_{e l}} \rho_{l} c_{p l} N_{i}(x) \psi_{j}^{n+1}(x) d x
$$

where $\Omega_{e s}$ and $\Omega_{e l}$ stands for the region of the solid- and the liquid-side sub-elements. Here, we introduce a reference coordinate $\xi$ for the enriched element and $\alpha$ for the sub-element (see Fig. 6). Both of the reference coordinate $\xi$ and $\alpha$ range from -1 to 1 . By using the Gaussian quadrature, the integration in the region $\Omega_{e s}$ is given by

$$
\begin{aligned}
\int_{\Omega_{e s}} \rho_{s} c_{p s} N_{i}(x) \psi_{j}^{n+1}(x) d x & =\rho_{s} c_{p s} \int_{-1}^{1} N_{i}(x(\alpha)) \psi_{j}^{n+1}(x(\alpha)) J(\alpha) d \alpha \\
& =\rho_{s} c_{p s} \int_{-1}^{1} N_{i}(\xi(\alpha)) \psi_{j}^{n+1}(\xi(\alpha)) J(\alpha) d \alpha \\
& =\rho_{s} c_{p s} \sum_{k=1}^{p} N_{i}\left(\xi\left(\alpha_{k}\right)\right) \psi_{j}^{n+1}\left(\xi\left(\alpha_{k}\right)\right) J\left(\alpha_{k}\right) W_{k}
\end{aligned}
$$

where $P$ is the number of the quadrature points, $\alpha_{k}$ is the coordinate of the $k$-th quadrature point and $W_{k}$ is the weight at the $k$-th quadrature point. $x(\alpha)$ and $\xi(\alpha)$ in Eq. (25) mean that both of the coordinate $x$ and $\xi$ are the function of the coordinate $\alpha . J(\alpha)$ is the Jacobian:

$$
J(\alpha)=\frac{\partial x}{\partial \alpha}
$$

The integration in the region $\Omega_{e l}$ can be obtained similarly. Since calculation of $N_{i}$ from the coordinate $x$ is almost impossible in multi-dimensional analyses which uses an unstructured mesh, $N_{i}(x)$ is replaced by $N_{i}(\xi)$ in Eq. (25). In this case, mapping from $\alpha$ to $\xi$ and mapping from $\alpha$ to $x$ must be given to get the integration. -1 and 1 on the coordinate $\alpha$ is translated into $\xi_{1}$ and $\xi_{I}$, respectively. Similarly, -1 and 1 on the coordinate $\alpha$ is translated into $x_{1}$ and $x_{I}$, respectively. From this information, the mappings are given by 


$$
\begin{aligned}
& \xi(\alpha)=N_{1}^{s u b}(\alpha) \xi_{1}+N_{I}^{s u b}(\alpha) \xi_{I} \\
& x(\alpha)=N_{1}^{s u b}(\alpha) x_{1}+N_{I}^{s u b}(\alpha) x_{I}
\end{aligned}
$$

where $N_{1}^{s u b}(\alpha)$ and $N_{I}^{s u b}(\alpha)$ are the shape functions shown in Fig. 6. The right hand side of Eqs. (27) and (28) are computable. We can calculate $\xi\left(\alpha_{k}\right)$ and $J\left(\alpha_{k}\right)$ from Eqs (26) to (28), and obtain the correct integration.

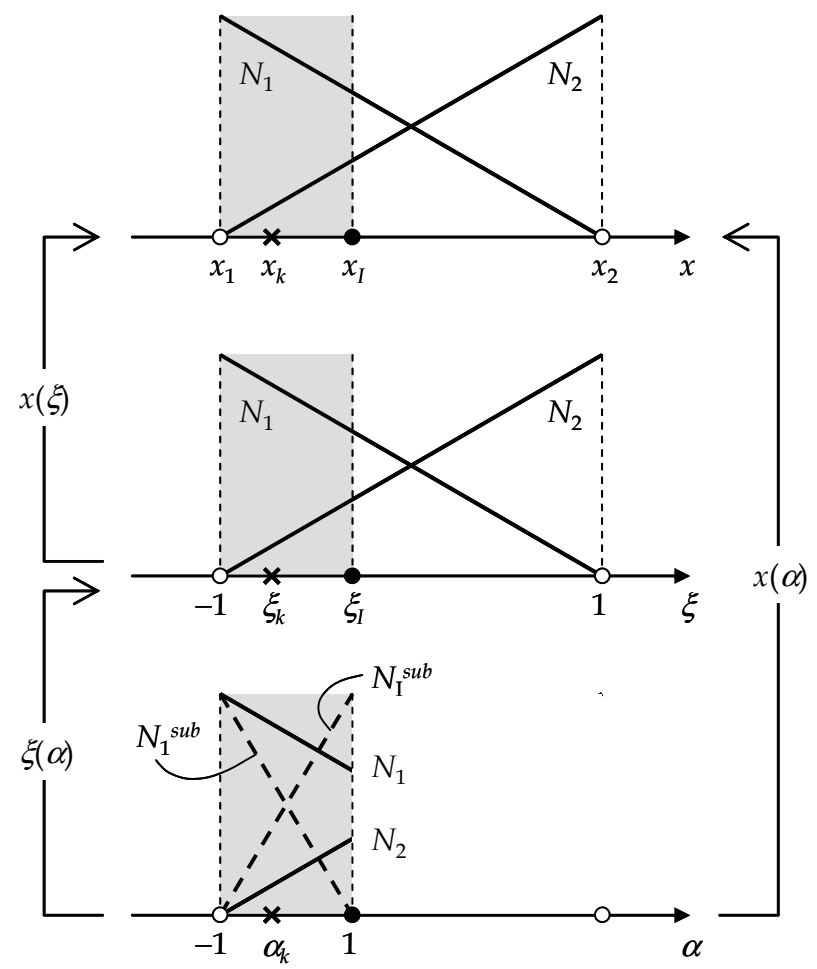

Fig. 6. Reference coordinates for enriched element

\subsection{Update of signed distance function}

The signed distance function is updated by the algorithm of the level set method (Osher \& Sethian, 1988; Sethian, 1999; Chessa et al., 2002). In this method, interface motion is described by the advective equation:

$$
\frac{\partial \phi}{\partial t}+F|\nabla \phi|=0
$$

where $F$ is the extension velocity to be described hereinafter. The Galerkin/Least Squares (GLS) method (Hughes et al., 1989) was applied to Eq. (29) for stabilization. The stabilized finite element equation is 


$$
\int_{\Omega} \delta \phi \frac{\partial \phi}{\partial t} d \Omega+\int_{\Omega} \delta \phi F|\nabla \phi| d \Omega+\sum_{e} \int_{\Omega_{e}}\left(\frac{F}{|\nabla \phi|} \nabla \delta \phi \cdot \nabla \phi\right) \tau_{e}\left(\frac{\partial \phi}{\partial t}+F|\nabla \phi|\right) d \Omega=0
$$

where $\delta \phi$ is the weight function and $\tau_{e}$ is the stabilization parameter. Formulation of the stabilization parameter was proposed by Tezduyar (1992). To solve Eq. (30), we first have to construct the extension velocity, which advects the signed distance function. The extension velocity has the characteristic that it be orthogonal to the signed distance function:

$$
\operatorname{sign}(\phi) \nabla F \cdot \nabla \phi=0
$$

Figure 7 shows the essential boundary condition for the extension velocity. $S$ is the intersection point between the solid-liquid interface and a perpendicular line from the enriched node P. Considering the orthogonal condition given by Eq. (31), it is obvious that the extension velocity at the node $\mathrm{P}$ is equal to the interface velocity at $\mathrm{S}$. Equation (31) is solved with taking the extension velocity at the enriched node as an essential boundary condition. The stabilized form of Eq. (31) is written as

$$
\int_{\Omega} \delta F \operatorname{sign}(\phi) \nabla F \cdot \nabla \phi d \Omega+\sum_{e} \int_{\Omega_{e}}(\nabla \delta F \cdot \nabla \phi) \tau_{e}(\nabla \phi \cdot \nabla F) d \Omega=0
$$

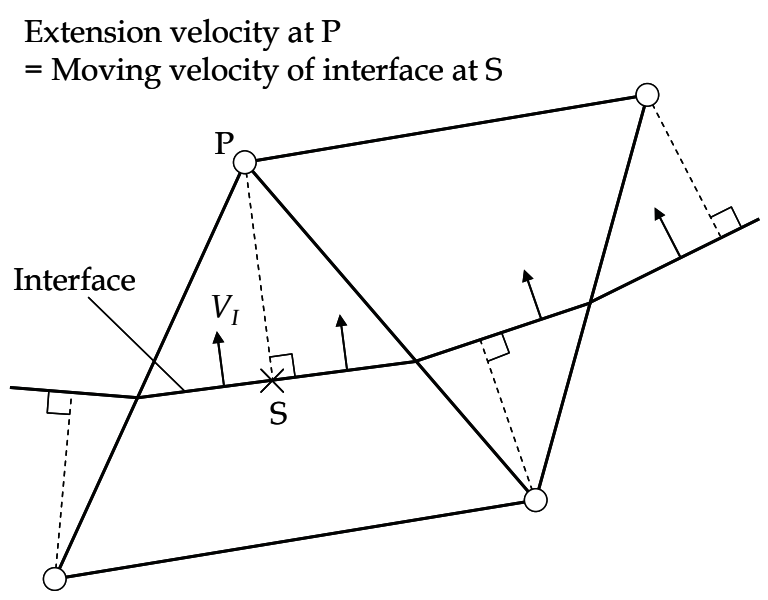

Fig. 7. Essential boundary condition of extension velocity

\subsection{Calculation of liquid flows}

Most problems in nuclear fuel area involve liquid flow. Liquid flow plays an important role in determining the shape of the solid-liquid interface. For example in injection casting or the process of high-level radioactive wastes disposal, flow of the molten mixture has an effect on performance of their process. In actual application of the numerical analysis, liquid flow and its effect on the behavior of the interface must be considered.

In our method, Eqs. (1) and (2) are solved by the velocity correction method (Ramaswamy, 1988). The Streamline-Upwind/Petrov-Galerkin (SUPG) scheme (Brooks \& Hughes, 1982) 
was applied to the convective term in Eq. (2). The effect of a buoyancy force was modelled by the Boussinesq approximation. To consider the condition that the flow velocity is 0 at the solid-liquid interface, the following essential boundary condition was applied to the enriched nodes which exist in the solid region:

$$
\mathbf{u}_{e b c}=-\frac{\phi_{l}}{\phi_{s}} \overline{\mathbf{u}}
$$

where $\mathbf{u}_{e b c}$ is the velocity at the enriched node in the solid region, $\phi$ is the length of the perpendicular line from the enriched node to the solid-liquid interface and $\overline{\mathbf{u}}$ is the velocity at the intersection between the perpendicular line and the element boundary (see Fig. 8). Equation (33) limits the velocity to 0 at the solid-liquid interface. $\mathbf{u}_{e b c}$ approaches 0 as the solid-liquid interface comes close to the enriched node in the solid region.

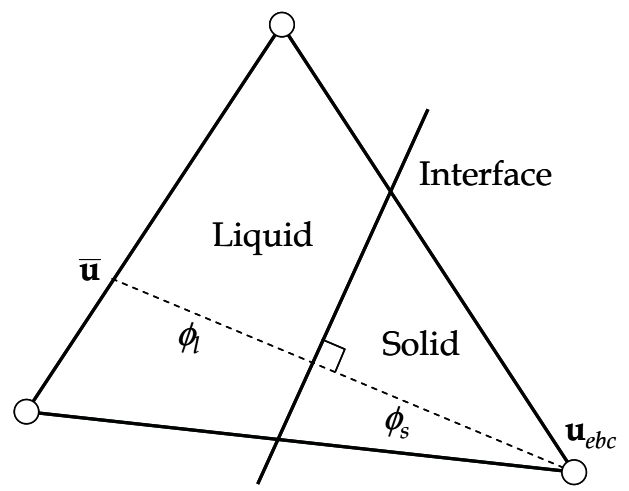

Fig. 8. Essential boundary condition of liquid-phase velocity

\subsection{Time-stepping procedure}

The time-stepping procedure is summarized below.

1. Construct the extension velocity $F$ from Eq. (32) under the essential boundary condition shown in Fig. 7. Equation (4) is used to give the essential boundary condition.

2. Calculate the signed distance function $\phi^{n+1}$ from Eq. (30).

3. Calculate the flow velocity $\mathbf{u}^{\mathrm{n}+1}$ from Eqs. (1) and (2) under the essential boundary condition given by Eq. (33).

4. Build the global matrix in Eq. (23) and solve for $T^{\mathrm{n}+1}$ by using the penalty method.

5. Return to step 1 and repeat the algorithm.

\section{Results and discussion}

\subsection{One-dimensional Stefan problem}

Numerical analysis of a one-dimensional melting problem using the X-FEM is now presented. The temperature at the one boundary is set to a value above the melting temperature, and the temperature at the other boundary is set to the melting temperature. This boundary condition keeps the temperature to the melting temperature in the whole 
solid region and causes move of the solid-liquid interface. This problem is the well-known one-phase Stefan problem. The exact solution describing the interface motion is given by

$$
x_{I}(t)=2 \eta \sqrt{\alpha_{l} t}
$$

where $x_{I}(t)$ is the interface position and $\alpha_{l}$ is the thermal diffusivity of the liquid phase. The constant $\eta$ satisfies the relationship:

$$
\frac{e^{-\eta^{2}}}{\operatorname{erf}(\eta)}=\frac{\eta L \sqrt{\pi}}{c_{l}\left(T_{l w}-T_{m}\right)}
$$

The temperature profile in the liquid region is given by

$$
T_{l}(x, t)=T_{l w}-\frac{T_{l w}-T_{m}}{\operatorname{erf}(\eta)} \operatorname{erf}\left(\frac{x}{2 \sqrt{\alpha_{l} t}}\right)
$$

The region which is $0.02 \mathrm{~m}$ in length was divided into the 10 finite elements. The initial temperature profile was given by the exact solution at $t=200 \mathrm{sec}$ (i.e. the solid-liquid interface was initially at $x=2.54 \times 10^{-3} \mathrm{~m}$ ). The boundary conditions were $T_{l w}=10^{\circ} \mathrm{C}$ at $x=0$ $\mathrm{m}$ and $T_{s w}=0{ }^{\circ} \mathrm{C}$ at $x=0.02 \mathrm{~m}$. The physical properties of the water were used in this analysis.

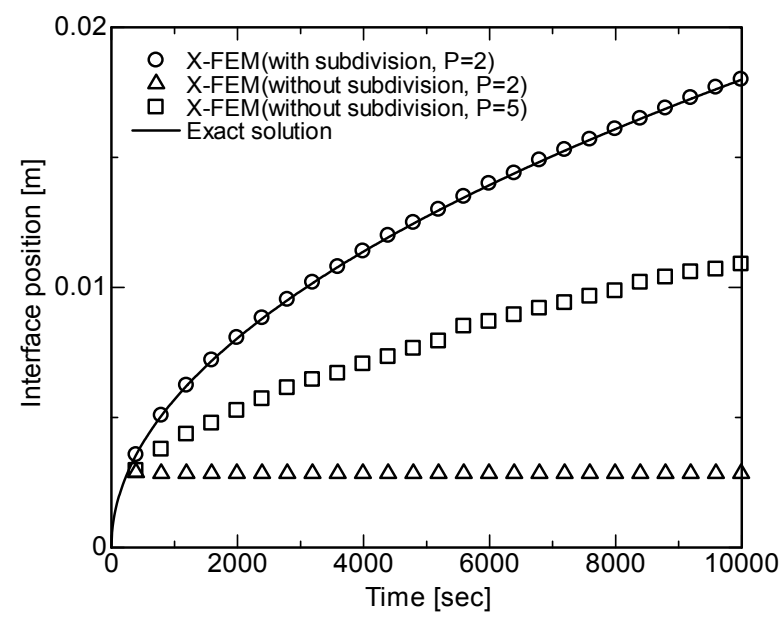

Fig. 9. Comparison of calculated interface position with exact solution in one-dimensional Stefan problem

Figure 9 shows the calculated interface positions and the exact solution as a function of time. The numerical analysis without subdivision of the enriched element was also conducted and its results are shown for comparison. The number of the quadrature point $P$ was taken as a parameter. As can be seen, the numerical analysis using the subdivision technique predicted the interface motion successfully. The relative error is within $1 \%$. When subdivision was not 
applied, the numerical analysis could not reproduce the interface motion even in the case of $P=5$. Figure 10 shows the temperature profiles at the four different times. The calculated temperature profiles agree with the exact solutions excellently. From this verification, it has been demonstrated that the X-FEM could predict the interface motion and the temperature profile accurately even in a fixed mesh.

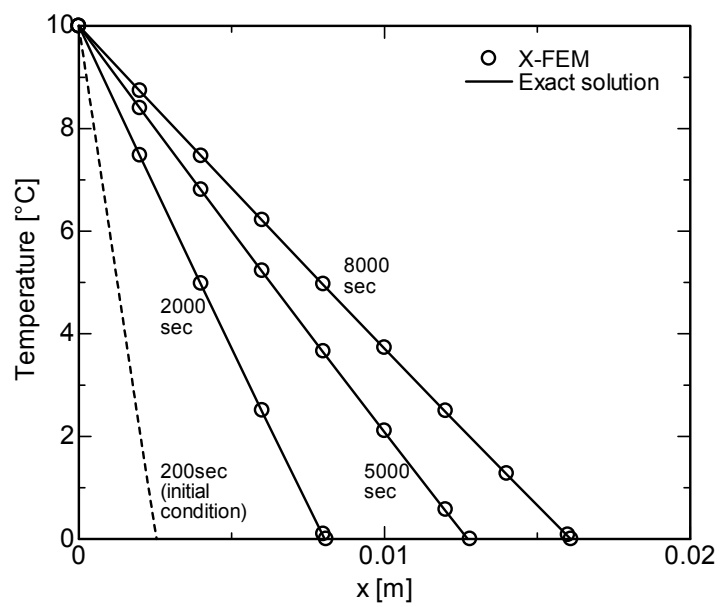

Fig. 10. Comparison of calculated temperature profile with exact solution in onedimensional Stefan problem

\subsection{Solidification in two-dimensional square corner}

The problem of solidification in a square corner was analyzed as a part of verification to multi-dimensional problems. An infinitely long prism is initially filled with a fluid at its melting temperature. Figure 11 shows the square-shaped cross section of the prism. The temperature on the peripheral surface of the prism is maintained at a certain value below the melting temperature so that solidification proceeds from the surface inward. There are no changes of the physical properties, and hence there is no convection in the liquid region. As shown in Fig. 11, the verification analysis was performed for the quarter region by taking the centerline of the prism as an adiabatic boundary. The analysis region was divided into the 400 triangle elements. The physical properties of the water were used in this analysis.

Figure 12 shows the temperature profile and the shape of the solid-liquid interface at the different times. The solid-liquid interface is denoted by the isoline of $\phi=0$. During the initial periods of solidification, the shape of the interface is close to square. As the time progresses, the curve near the diagonal gradually flattens. The interface position along the centerline, i.e. the adiabatic boundary, and along the diagonal line is shown against time in Fig. 13, respectively. The approximate solutions derived by Allen \& Severn (1962), Crank \& Gupta (1975), Lazaridis (1970) and Rathjen \& Jiji (1971) are plotted for comparison. The results of the X-FEM agree with some of the approximate solutions very well. The difference between the numerical results and some of the solutions seems to be due to the approximations used in the previous researches. 

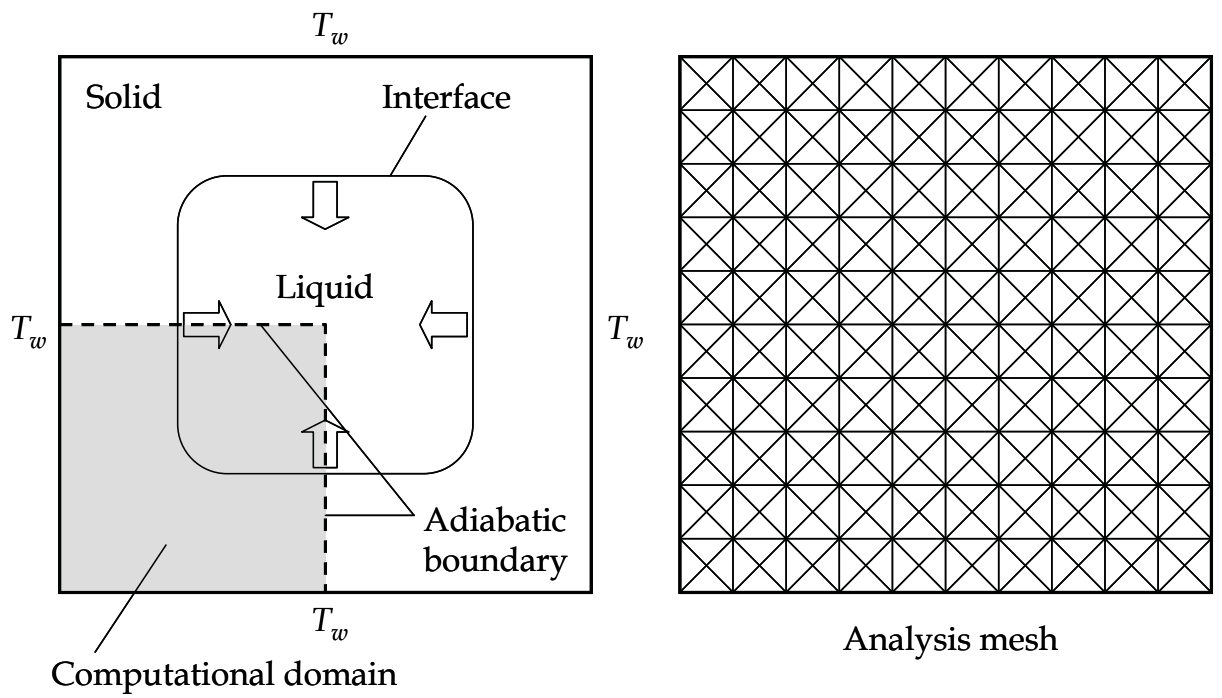

Fig. 11. Computational domain for solidification in two-dimensional square corner

$\tau:$ dimensionless time $=t \lambda /(\rho c \mathrm{H})$

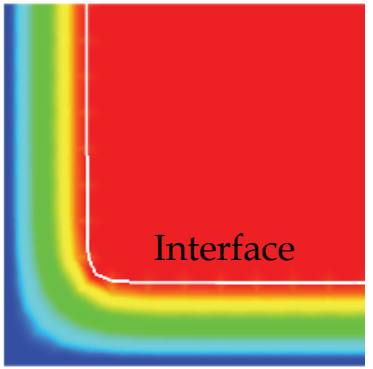

$\tau=0.042$

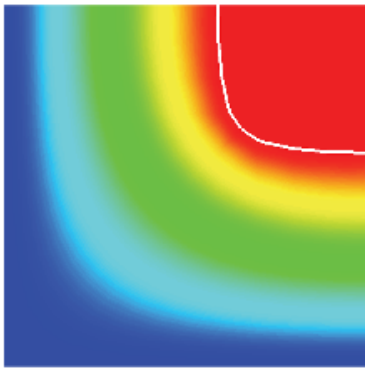

$\tau=0.304$

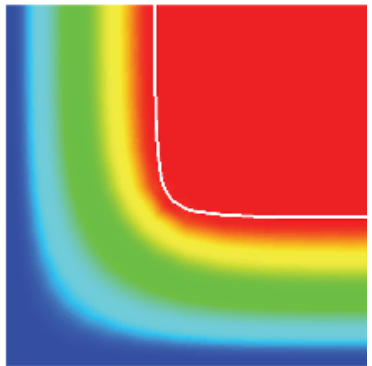

$\tau=0.147$

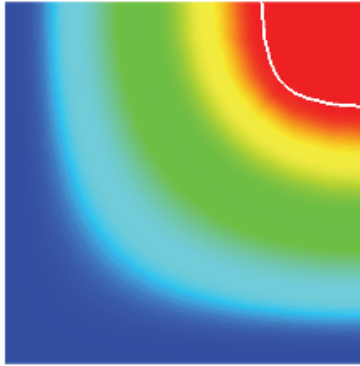

$\tau=0.419$

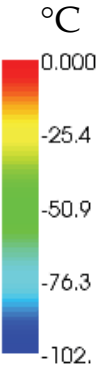

Fig. 12. Calculated solid-liquid interface and temperature in two-dimensional square corner 


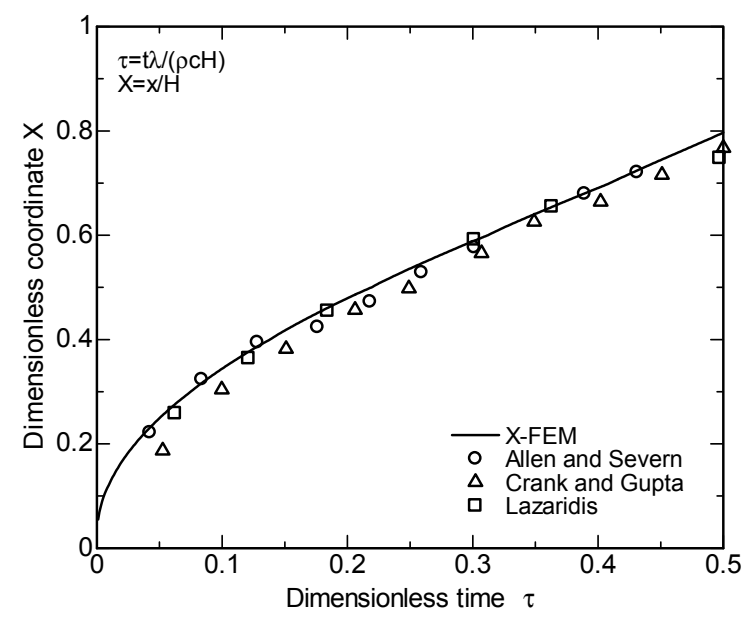

(a) Interface position along center line

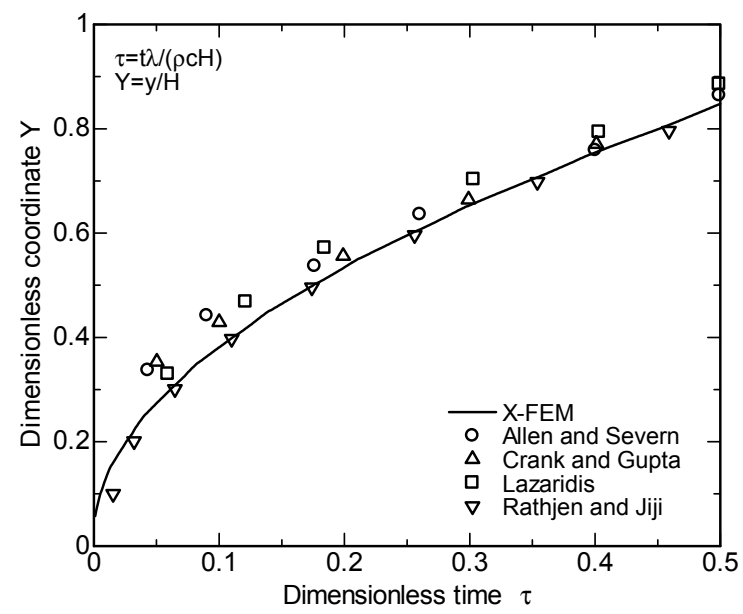

(b) Interface position along diagonal line

Fig. 13. Comparison of calculated interface position with existing solutions in twodimensional square corner

\subsection{Melting of gallium in two-dimensional cavity}

Gau \& Viskanta (1986) conducted the experiment on melting of pure gallium. Their experiment was analyzed to confirm applicability of our numerical analysis method to the problem involving liquid flow. The two-dimensional cavity which is $0.04445 \mathrm{~m}$ in height and $0.0889 \mathrm{~m}$ in width (see Fig. 14) is initially filled with pure solid gallium at its melting temperature $\left(29.78{ }^{\circ} \mathrm{C}\right)$. At $t=0$, the temperature on the left-hand wall is raised to $38^{\circ} \mathrm{C}$. On 
the other hand, the temperature on the right-hand wall is kept at the melting temperature. The upper and the lower walls are insulated. As time increases, melting proceeds from the left-hand vertical wall rightward. In this problem, natural convection occurs because of the existence of the gravitational force and the density change. The cavity was divided into the 800 triangle elements.

Figure 15 shows the calculated interface, temperature profile and streamlines. It can be seen that the solid-liquid interface inclined rightward from the vertical axis. Since the temperature gradient near the bottom of the liquid region is relatively low, the interface at the bottom moves more slowly. Thus, natural convection plays an important role in determining the shape of the solid-liquid interface. As shown in Fig. 16, the calculated interface shape shows good agreement with the experimental results by Gau \& Viskanta. Applicability to the actual problems in the nuclear fuel area was demonstrated through this analysis. The slight difference is probably due to the uncertainty on the temperature at the right-hand wall in the experiment, which is mentioned by Gau \& Viskanta (1986), and the anisotropic thermal conductivity of the pure gallium (Cubberley, 1979).

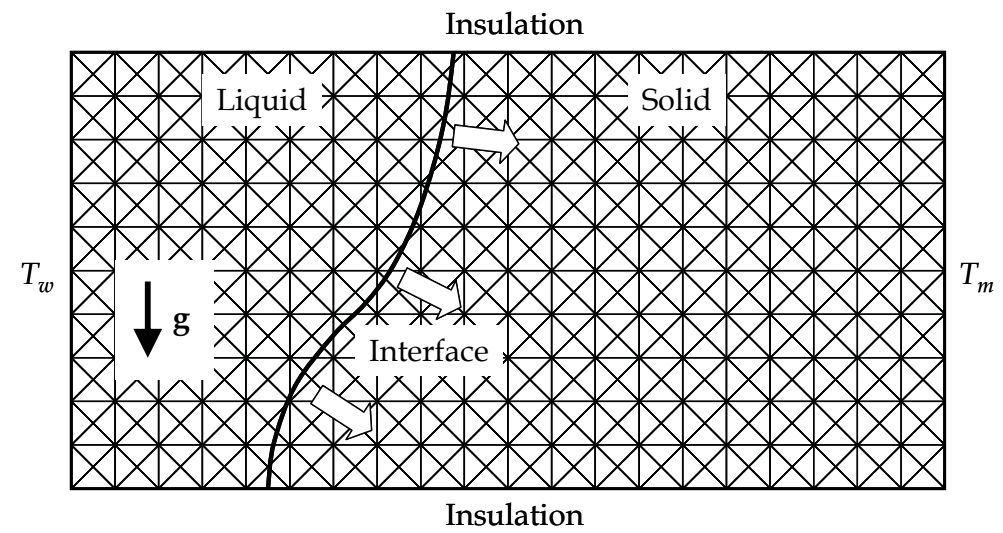

Fig. 14. Computational domain for melting of pure gallium in two-dimensional cavity
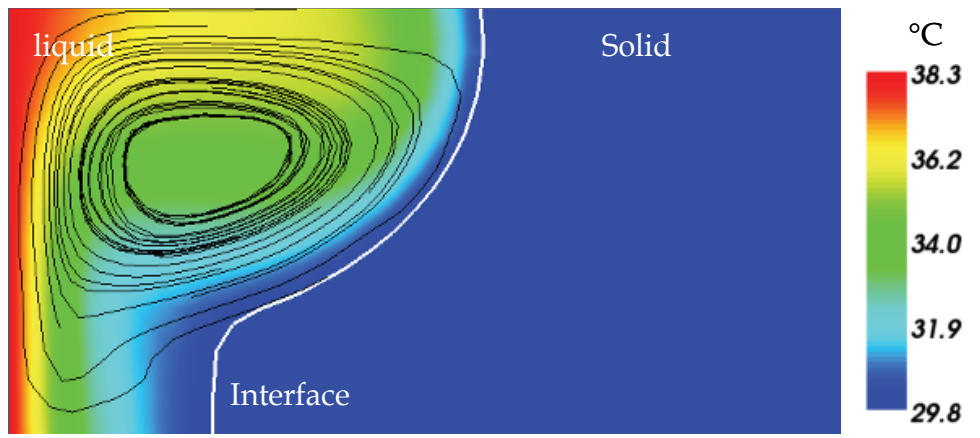

Fig. 15. Calculated solid-liquid interface, temperature and streamlines in two-dimensional gallium melting problem 


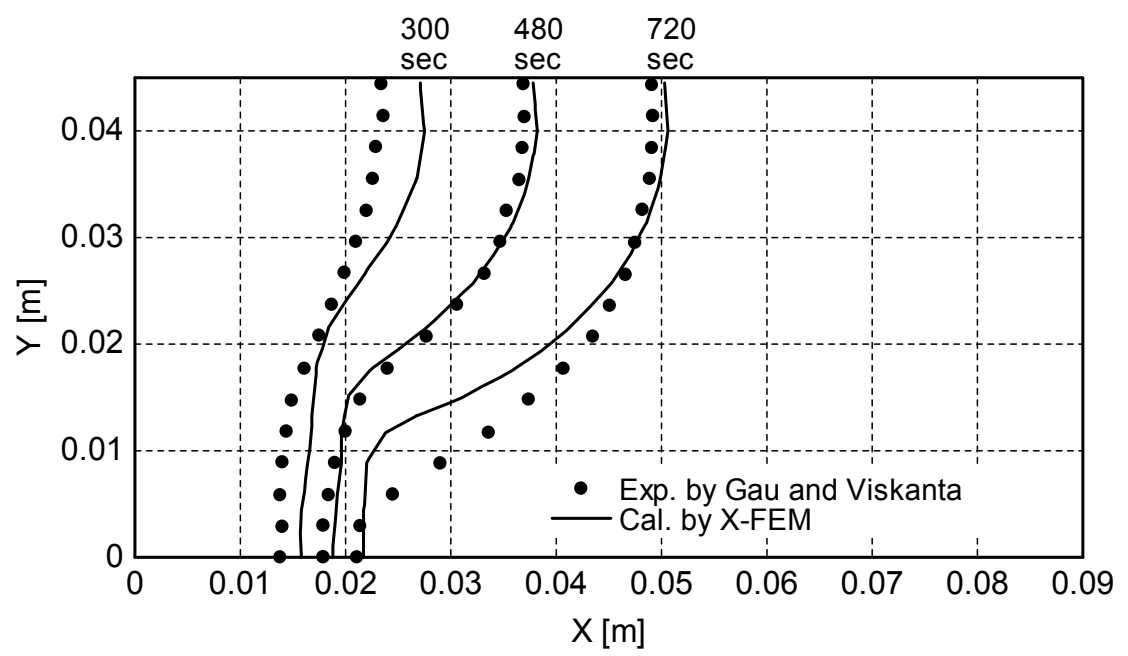

Fig. 16. Comparison of calculated interface position with experimental data in twodimensional gallium melting problem

\section{Conclusion}

In this study, the X-FEM was applied to melting/solidification problems. The method uses the enriched finite element interpolation to represent the discontinuous temperature gradient in the element crossed by the solid-liquid interface. Construction of the enriched finite element interpolation and the finite element equation using it was discussed. We formulated the numerical integration for the enriched element. The method to solve problems involving liquid flows was also developed in the present work. From the numerical analysis of the one-dimensional Stefan problem, it has been demonstrated that the X-FEM could predict the interface motion and the temperature profile accurately even in a fixed mesh. As a part of verification to multi-dimensional problems, the solidification in the two-dimensional square corner was also analyzed. The numerical results on the interface position showed good agreement with some of the approximate solutions. In the analysis of melting of pure gallium, the shape of the solid-liquid interface was reproduced successfully. These verifications indicate that our numerical analysis method is physically correct and applicable to the multi-dimensional problems involving liquid flow. This method makes it possible to evaluate melting/solidification processes appearing in injection casting and the process of high-level radioactive wastes disposal, etc. The future work is to develop a mass transfer model to simulate multi-component dissolution/ precipitation phenomena.

\section{References}

Allen, D. N. de G. \& Severn, R. T. (1962). The Application of Relaxation Methods to the Solution of Non-elliptic Partial Differential Equations III. Q. Jl Mech. Appl. Math., Vol.15, pp. 53-62, 1962. 
Brooks, A. N. \& Hughes, T. J. R. (1982). Streamline-Upwind/Petrov-Galerkin Formulations for Convection Dominated Flows with Particular Emphasis on the Incompressible Navier-Stokes Equations. Comput. Meth. Appl. Mech. Engrg., Vol.32, pp. 199, 1982.

Chessa, J. \& Belytschko, T. (2003). The Extended Finite Element Method for Two-phase Fluids. ASME J. Appl. Mech., Vol.70, pp. 10-17, 2003.

Chessa, J.; Smolinski, P. \& Belytschko, T. (2002). The Extended Finite Element Method (XFEM) for Solidification Problems. Int. J. Numer. Meth. Engng., Vol.53, pp. 1959-1977, 2002.

Chessa, J.; Wang, H. \& Belytschko, T. (2003). On Construction of Blending Elements for Local Partition of Unity Enriched Finite Element Methods. Int. J. Numer. Meth. Engng., Vol.57, pp. 1015-1038, 2003.

Comini, G.; Guidice, S. Del; Lewis, R. W. \& Zienkiewicz, O. C. (1974). Finite Element Solution on Non-Linear Heat Conduction Problems with Special Reference to Phase Change. Int. J. Numer. Meth. Engng., Vol.8, pp. 613-624, 1974.

Crank, J. \& Gupta, R. S. (1975). Isotherm Migration Method in Two Dimensions. Int. J. Heat Mass Transfer, Vol.18, pp. 1101-1107, 1975.

Cubberley, W. H. Metal Handbook-Properties and Selection: Nonferrous Alloys and Pure Metals, 9th edn., American Society of Metals, Metals Park, OH, pp. 736-737, 1979.

Funasaka, H. \& Itoh, M. (2007). Perspective and Current Status on Fuel Cycle System of Fast Reactor Cycle Technology Development (FaCT) Project in Japan. Proceedings of the GLOBAL 2007, Boise, Idaho, USA, Sep 9-13, 2007.

Gau, C. \& Viskanta, R. Melting and Solidification of a Pure Metal on a Vertical Wall. Trans. ASME J. Heat Transfer, Vol.108, pp. 174-181, 1986.

Hughes, T. J. R.; Franca, L. P. \& Hulbert, G. M. (1989). A New Finite Element Formulation for Computational Fluid Dynamics: VIII. The Galerkin/Least-Squares Method for Advective-Diffusive Equations. Comput. Meths. Appl. Mech. Engrg., Vol.73, pp. 173189, 1989.

Jaeger, M. \& Carin, M. (1994). The Front-Tracking ALE Method: Application to a Model of the Freezing of Cell Suspensions. J. Comp. Phys., Vol.179, pp. 704-735, 1994.

Kofuji, H. (2010). Electro-Deposition Behavior of Minor Actinides with Liquid Cadmium Cathodes. IOP Conference Series; Materials Science and Engineering, vol.9, pp. 0120101-012010-8, 2010.

Kozaka, T. \& Tominaga, S. (2005). Development of the Vitrification Technology at TVF. JNC Technical Review, JNC TN1340 2005-002, pp. 35-39, 2005.

Lazaridis, A. (1970). A Numerical Solution of the Multidimensional Solidification (or Melting) Problem. Int. J. Heat Mass Transfer, Vol.13, pp. 1459-1477, 1970.

Lynch, D. R. \& O'Neill, K. (1981). Continuously Deforming Finite Elements for the Solution of Parabolic Problems, with and without Phase Change. Int. J. Numer. Meth. Engng., Vol.17, pp. 81-96, 1981.

Merle, R. and Dolbow, J. (2002). Solving Thermal and Phase Change Problems with the Extended Finite Element Method. Comp. Mech., Vol.28, pp. 339-350, 2002.

Moës, N.; Dolbow, J. \& Belytschko, T. (1999). A Finite Element Method for Crack Growth without remeshing. Int. J. Numer. Meth. Engng., Vol.46, pp. 131-150, 1999.

Murray, W. D. \& Landis, F. (1959). Numerical and Machine Solutions of Transient HeatConduction Problems Involving Melting or Freezing. ASME J. Heat Transfer, Vol.81, pp. 106-112, 1959. 
Osher, S. \& Sethian, J. A. (1988). Propagation of Fronts with Curvature Based Speed: Algorithms Based on Hamilton-Jacobi Formulations. J. Comp. Phys., Vol.79, pp. $12-$ 49, 1988.

Ramaswamy, B. (1988). Finite Element Solution for Advection and Natural Convection Flows, Comput. Fluids, Vol.16, pp. 349, 1988.

Rathjen, K. A. \& Jiji, L. M. (1971). Heat Conduction with Melting or Freezing in a Corner. Trans. ASME J. Heat Transfer, Vol.93, pp. 101-109, 1971.

Rolph III, W. D. \& Bathe, K. J. (1982). An Efficient Algorithm for Analysis of Nonlinear Heat Transfer with Phase Change. Int. J. Numer. Meth. Engng., Vol.18, pp. 119-134, 1982.

Sagayama, Y. (2007). Launch of Fast Reactor Cycle Technology Development Project in Japan. Proceedings of the GLOBAL 2007, Boise, Idaho, USA, Sep 9-13, 2007.

Sampath, R. \& Zabaras, N. (1999). An Object Oriented Implementation of a Front Tracking Finite Element Method for Directional Solidification Processes. Int. J. Numer. Meth. Engng., Vol.44, pp. 1227-1265, 1999.

Sethian, J. A. (1999). Level Set Methods and Fast Marching Methods. Evolving Interfaces in Computational Geometry, Fluid Mechanics, Computer Vision, and Materials Sciences. Cambridge Monographs on Applied and Computational Mathematics, Cambridge University Press, Cambridge, 1999.

Tezduyar, T. E. (1992). Stabilized Finite Element Formulations for Incompressible Flow Computations. Adv. Appl. Mech., Vol.28, pp. 1-44, 1992. 


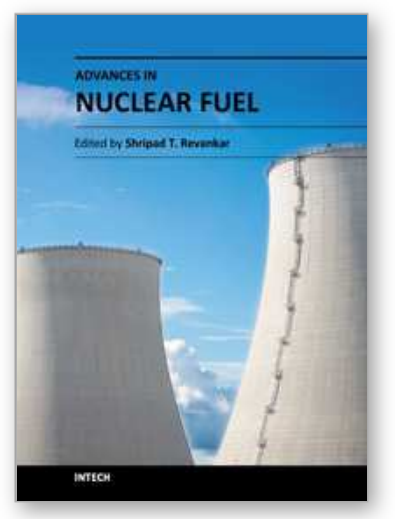

\author{
Advances in Nuclear Fuel \\ Edited by Dr. Shripad T. Revankar
}

ISBN 978-953-51-0042-3

Hard cover, 174 pages

Publisher InTech

Published online 22, February, 2012

Published in print edition February, 2012

Worldwide there are more than 430 nuclear power plants operating and more plants are being constructed or planned for construction. For nuclear power to be sustainable the nuclear fuel must be sustainable and there should be adequate nuclear fuel waste management program. Continuous technological advances will lead towards sustainable nuclear fuel through closed fuel cycles and advance fuel development. This focuses on challenges and issues that need to be addressed for better performance and safety of nuclear fuel in nuclear plants. These focused areas are on development of high conductivity new fuels, radiation induced corrosion, fuel behavior during abnormal events in reactor, and decontamination of radioactive material.

\title{
How to reference
}

In order to correctly reference this scholarly work, feel free to copy and paste the following:

Akihiro Uchibori and Hiroyuki Ohshima (2012). Numerical Analysis of Melting/Solidification Phenomena Using a Moving Boundary Analysis Method X-FEM, Advances in Nuclear Fuel, Dr. Shripad T. Revankar (Ed.), ISBN: 978-953-51-0042-3, InTech, Available from: http://www.intechopen.com/books/advances-in-nuclearfuel/numerical-analysis-of-melting-solidification-phenomena-using-a-moving-boundary-analysis-method-x-fem

\section{INTECH}

open science | open minds

\section{InTech Europe}

University Campus STeP Ri Slavka Krautzeka 83/A 51000 Rijeka, Croatia Phone: +385 (51) 770447

Fax: +385 (51) 686166 www.intechopen.com

\section{InTech China}

Unit 405, Office Block, Hotel Equatorial Shanghai

No.65, Yan An Road (West), Shanghai, 200040, China 中国上海市延安西路65号上海国际贵都大饭店办公楼 405 单元 Phone: +86-21-62489820

Fax: +86-21-62489821 
(C) 2012 The Author(s). Licensee IntechOpen. This is an open access article distributed under the terms of the Creative Commons Attribution 3.0 License, which permits unrestricted use, distribution, and reproduction in any medium, provided the original work is properly cited. 